\title{
Atractocybeloides, eine kleinwüchsige Trilobitengattung aus baltoskandischen Geschieben und ihrem Anstehenden
}

\author{
Hans-Hartmut Krueger ${ }^{1}$
}

Mit 6 Abbildungen und 3 Tafeln

\section{Zusammenfassung}

\begin{abstract}
Aus mittel- bis oberordovizischen Geschieben werden fünf Taxa der kleinwüchsigen Trilobitengattung Atractocybeloides vorgestellt, davon die beiden neuen Arten Atractocybeloides nebeni $\mathrm{n}$. sp. und Atractocybeloides oepiki n. sp. Die Gattung wurde 1991 an Fragmenten aufgestellt. Sie hat sich in der Aseri-Stufe aus Cybele entwickelt. Neue Funde und das reichhaltige Material aus der Sammlung Rhebergen, in der auch fast vollständige Panzerhemden von $A$. berneri enthalten sind, ermöglichten eine Rekonstruktion. Weiterhin wird die aus Norwegen beschriebene Art Atractopyge gracilis der Gattung Atractocybeloides zugeordnet. Große Ähnlichkeiten bestehen zur ungefähr gleichaltrigen nordamerikanischen kleinwüchsigen Gattung $C y$ beloides.
\end{abstract}

Schlüsselwörter: Trilobita, Atractocybeloides, Geschiebe, Ordovizium.

\begin{abstract}
Five taxa of the small trilobite genus Atractocybeloides are presented from Middle to Late Ordovician erratic boulders, including the new species Atractocybeloides nebeni $\mathrm{n}$. sp. and Atractocybeloides oepiki $\mathrm{n}$. sp. The genus was errected in 1991 on the basis of fragmentary material. It evolved from Cybele during Aseri-zone times. A reconstruction is made possible due to new finds and the rich material from the Rhebergen collection, including nearly complete cuticles of $A$. berneri. Furthermore, Atractopyge gracilis from Norway is assigned to Atractocybeloides. Strong similarities exist to the roughly coeval, small trilobite genus Cybeloides, which is known from North America.
\end{abstract}

Key words: Trilobita, Atractocybeloides, erratic boulders, Ordovician.

\section{Einleitung}

Durch ihren kleinen Wuchs und die seltenen, spärlichen Reste in bestimmten oberordovizischen Geschieben blieb die Gattung Atractocybeloides aus Baltoskandia und seinen Geschieben bis vor zehn Jahren unerkannt. In den achtziger Jahren fand der Präparator L. Berner, Berlin, ein kleines Cranidium einer Cybele-Art in einem verkieselten Kalk (Backsteinkalk). Dieses Cranidium wich vom typischen Aussehen einer Cybele oder Atractopyge ab. Es fehlt ein Präglabellarfeld, der Nackenring trägt einen Stachel und es sind große Wangenstachel vorhanden. Erst weitere Funde aus den verkieselten Geröllen aus den Deckschichten des 2. Lausitzer Braunkohlenflözes sowie von Sylt ließen eine neue Trilobitengattung erkennen. Die neue Gat- tung vereinigt auf ihrem Cranidium Merkmale von Cybele, Atractopyge und Cybeloides. 1991 wurde die neue Gattung Atractocybeloides mit zwei neuen Arten, $A$. berneri und $A$. vonhachti, beschrieben (Krueger 1991). Nikolaisen beschrieb 1961 unter anderen einige AtractopygeArten aus dem Oslo-Gebiet. Eine neue, von ihm aufgestellte Art, Atractopyge gracilis, zeichnete sich durch große, lange Wangenstachel und dem Fehlen eines Präglabellarfeldes aus. Nikolaisen nahm an, dass bei allen neun ihm zur Verfügung stehenden Cranidien das Präglabellarfeld schon vor der Einbettung abgebrochen war. Diese Art aus Norwegen wird jetzt zu Atractocybeloides gestellt und ist der jüngste Vertreter dieser Gattung. Fünf Arten werden vorgestellt und beschrieben, wovon zwei Arten neu sind (Abb. 1). Neue Funde und das reichhaltige Material von

\footnotetext{
${ }^{1}$ Museum für Naturkunde der Humboldt Universität, Institut für Paläontologie, Invalidenstr. 43, D-10115 Berlin, Germany. Erhalten April 2002, angenommen Juni 2002
} 


\begin{tabular}{|c|c|c|c|}
\hline$E$ & & A. gracilis & 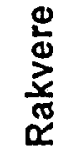 \\
\hline$D_{111}$ & & & 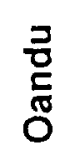 \\
\hline $\begin{array}{l}\mathrm{D}_{\| \beta} \\
\mathrm{D}_{\| \alpha}\end{array}$ & 1 & $\left.\right|_{\text {A. vonhachti }}$ & $\frac{\infty}{\overline{0}}$ \\
\hline$D_{1}$ & $\begin{array}{l}1 \\
0\end{array}$ & & 文 \\
\hline $\begin{array}{l}\mathrm{C}_{\| 1 \beta \beta} \\
\mathrm{C}_{\| l \alpha}\end{array}$ & $\overline{0}$ & A. berneri & 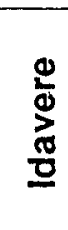 \\
\hline $\begin{array}{l}\mathrm{C}_{\| \beta} \\
\mathrm{C}_{\| \alpha}\end{array}$ & $\frac{0}{0}$ & A. oepiki n.sp. & 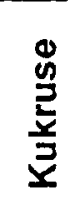 \\
\hline $\begin{array}{l}C_{l c \beta} \\
C_{l c \alpha}\end{array}$ & & & $\frac{\vec{z}}{\frac{\partial}{d}}$ \\
\hline $\begin{array}{l}C_{l b \gamma} \\
C_{I b \beta} \\
C_{l b \alpha}\end{array}$ & & $\begin{array}{c}\text { Atractocybeloides } \\
\text { nebeni n.sp. }\end{array}$ & 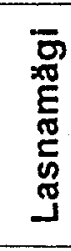 \\
\hline $\mathrm{C}_{\text {la } \beta}$ & Cybele & & : \\
\hline $\begin{array}{l}\mathrm{B}_{\| I \mid \gamma} \\
\mathrm{B}_{\| I \mid \beta} \\
\mathrm{B}_{\|\| \alpha}\end{array}$ & Cybele & & $\stackrel{\frac{\pi}{2}}{\stackrel{\underline{C}}{3}}$ \\
\hline
\end{tabular}

Abb. 1. Stratigraphische Verbreitung von Arten von Atractocybeloides in baltoskandischen Geschieben aus dem Mittleren und Oberen Ordovizium. 
A. berneri in der Sammlung Rhebergen, in der Cranidien, Freiwangen, Thoraxe sowie Pygidien vorhanden sind, empfahlen, die 1991 aufgestellten Arten $A$. berneri und $A$. vonhachti neu zu beschreiben.

\section{Material}

Diese relativ kleinwüchsige Trilobitengattung, die nur ca. $10 \mathrm{~mm}$ breit und $30 \mathrm{~mm}$ lang ist, kommt in bestimmten baltoskandischen Geschieben sehr selten vor. Kalkgeschiebe aus der Lasnamägi-Stufe, die den bisher ältesten Vertreter dieser Gattung geliefert haben, sind im ehemaligen Vereisungsgebiet südlich der Ostsee verbreitet, aber nicht häufig. Atractocybeloides und die nordamerikanische Gattung Cybeloides sind sich in ihrem kleinen Wuchs und im Panzer sehr ähnlich. Cybeloides-Reste sind in den mittelordovizischen Schichten Virginias und Kanadas als Verkieselungen erhalten und können aus ihrem umhüllenden Gestein herausgelöst werden. Günstige Bedingungen lieferten die Kiesgruben bei Wilsum im deutsch-niederländischen Grenzgebiet in den siebziger und achtziger Jahren des letzten Jahrhunderts, wo ein großer Anteil der anfallenden Geschiebe als teilweise verwitterter, ehemaliger verkieselter Kalk, der aus dem oberen Teil der Idavere- und Johvi Stufe $\mathrm{C}_{\mathrm{II} /} \mathrm{D}^{-} \mathrm{D}_{\mathrm{I}}$ stammt, aufgesammelt werden konnte. Bei Tausenden von Geschieben dieses Typs konnte Herr Rhebergen neben vielen verschiedenen Trilobiten-, Brachiopoden- und Gastropodengattungen auch eine Vielzahl von Exemplaren von Atractocybeloides berneri bergen, die es ermöglichen, eine Rekonstruktion dieser Gattung zu erstellen. Die Präparation ist wegen der Kleinwüchsigkeit dieser Trilobitengattung und ihrem Vorkommen in verkieselten bis stark verwitterten Geschieben sehr schwierig. Die Gesteinsbeschaffenheit kann von sehr hart zu pulverförmiger Substanz innerhalb von wenigen Millimetern wechseln. Die Fossilreste sind als Steinkerne erhalten. Nur bei günstiger Erhaltung kann vom Abdruck die Schalenoberfläche als Abguss gewonnen werden.

Die Stücke befinden sich in folgenden Museen:

- Museum für Naturkunde Berlin (MB.T.)

- Rijksmuseum van Geologie en Mineralogie Leiden, Niederlande (RGM)

- Palaeontological Museum Oslo, University of Oslo, Norway (P.M.O.)

- Archiv für Geschiebekunde Hamburg G 113/1

\section{Systematische Beschreibungen}

Familie Encrinuridae Angelin, 1854

Subfamilie Cybelinae Holliday, 1942

Gattung Atractocybeloides Krueger, 1991

Typusart: Atractocybeloides berneri Krueger, 1991.

\section{Atractocybeloides nebeni $\mathbf{n}$. sp.}

Tafel 1: 1-4, Abb. 2

Holoty pus: 1 Cranidium, MB.T.4492.1

Locus typicus: Röbel, Mecklenburg.

Stratum typicum: Lasnamägi-Stufe, $\mathrm{C}_{\mathrm{Ib} \gamma}$, Folkeslundakalk, tiefes Viru.
Derivatio nominis: nach dem Geschiebesammler Walter Neben.

Material: 1 Cranidium in Schalenerhaltung.

Maße (in $\mathrm{mm}$ ):

Glabella, größte Breite bei L $1 \quad 6,0$

Glabella, kleinste Breite Frontallobus 5,5

Glabella + Occipitalring, Länge $\quad 9,0$

Occipitalring, Breite $\quad 6,5$

Cranidium, Breite $\approx 30,2$

Diagnose: Frontallobusvorderkante in der Mitte gerade, zu den Seiten abgeschrägt, in den Fossulagruben endend. Mediangrube vorhanden. Glabella mit sechs paarig angeordneten Tuberkeln, Glabellarfurchen leicht nach vorn und nach außen gerichtet. Occipitalring median mit Stachel. Festwangen breit, Augen gestielt, Wangenstachel kräftig und lang.

Beschreibung: Cranidium flach gewölbt, nur zu den Seiten steiler nach unten zu den Wangenstacheln gebogen. Glabella nach vorn und zu den Seiten gleichmäßig flach gewölbt, am geraden Mittelteil des Frontallobus vorn und an den abgeschrägten Seiten steiler gebogen. Occipitalring von der Glabella durch eine bogenförmige, nach vorn gerichtete, flache Furche abgesetzt, an beiden Seiten in verbreiterte, tiefe Gruben mündend. Median einen kurzen, dornartigen, nach oben gerichteten Stachel tragend. L1-Loben kräftig und rundlich entwickelt, von den L2-Loben, die leicht nach vorn gerichtet sind, durch tiefe, dreieckig angelegte Lateralfurchen getrennt. Kräftiger entwickelte L3-Loben leicht nach vorn gerichtet, zu L2 durch tiefe, aber schmale Furchen abgesetzt. Frontallobus mit relativ großer, mitteltiefer Mediangrube. Glabellaoberfläche mit sechs paarig angelegten Tuberkeln bedeckt, die größten oberhalb der Mediangrube und in den Ecken zwischen geradem Vorderrand und abgeschrägtem Seitenrand. Hinter dem Vorderrand und der Mediangrube Gruppe von zehn kleinen, dicht liegenden Tuberkeln besetzt. Glabella und Festwangen durch gerade nach vorn verlaufende, sehr flache Dorsalfurchen getrennt, in Höhe der L3-Furchen von den schräg nach hinten gerichteten, gut entwickelten Augenleisten begrenzt (Tafel 1: 1). Festwangen breit, zu den Augenstielen leicht erhöht und nach außen zur Wangenstachelbasis steil abfallend. Festwangen-Vorderteil zwischen Augenleisten und Gesichtsnaht schmal und zum Frontallobus durch gut ausgebildete Fossulagruben begrenzt. Hinterrand der Festwangen im Innenteil fast gerade, erst in Hö- 


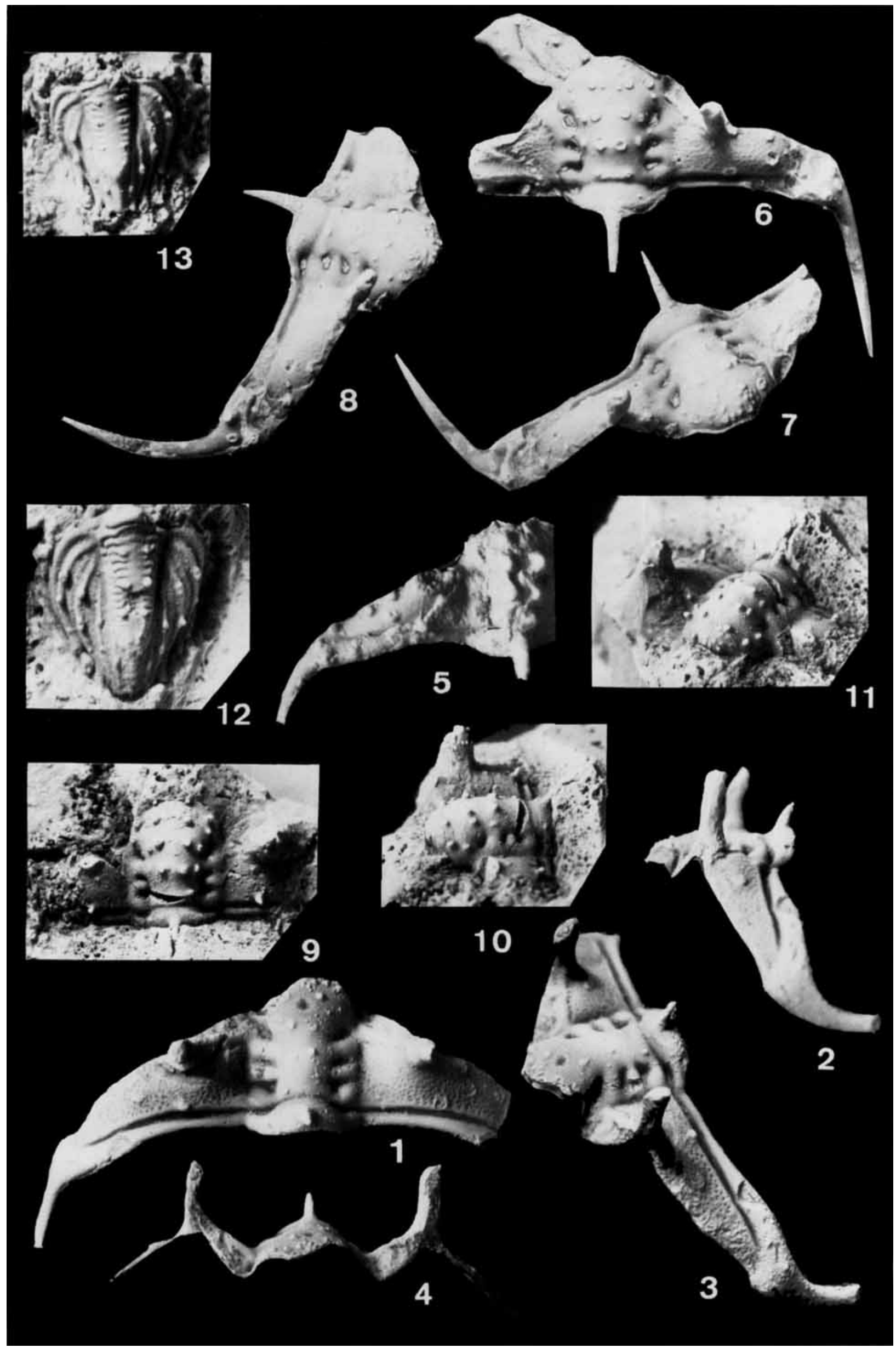


he der Augen in leichtem Bogen nach hinten schwingend, in der Wangenstachelbasis endend. Hinterrandfurche tief und halb so breit wie der Hinterrand in Höhe der Augen. Im Außenteil Hinterrand breiter, um in die gut ausgebildete Wangenstachelbasis überzugehen. Wangenstacheln kräftig und im Querschnitt flach oval. Am Anfang leicht nach außen schwingend, um dann gerade nach hinten und oben zu verlaufen. Länge nicht bekannt, aber es ist anzunehmen, daß sie der Länge der Glabella entspricht. Augenstiele gerade und schlank, ihre Höhe entspricht ungefähr drei Viertel der Glabellalänge. Gesichtsnaht von den Fossulagruben zu den Augen leicht bogenförmig verlaufend, um dann leicht nach hinten abgeschrägt zur Wangenstachelbasis zu gehen. Torulartuberkel schwach entwickelt, wogegen der Posttorulartuberkel kräftig und mittelhoch ist, ebenso die Tuberkeln, die zwischen Augenstiel und Wangenstachelbasis liegen. Ein mittelgroßer Tuberkel liegt auf der Augenleiste. Zwei bis drei sehr kleine Tuberkeln auf der Wangenstachelbasis. Schalenoberfläche der Festwangen dicht mit Grübchen bedeckt und die Glabella fein granuliert (Abb. 2).
Unterschiede: $A$. nebeni n. sp. unterscheidet sich von den jüngeren Arten durch die spärliche Tuberkulierung auf der Glabella, dem senkrecht stehenden dornartigen Stachel auf dem Occipitalring und dem abgeflachten, kräftigen Wangenstachel (Tafel 1: 1-4).

B e merkungen: Atractocybeloides nebeni $\mathrm{n}$. sp. besitzt noch Merkmale von Cybele: die Mediangrube und die Ausbildung der Festwangen mit den auf diesen vorhandenen Tuberkeln.

Vorkommen: Die Fauna setzt sich wie folgt zusammen: Asaphus (Neoasaphus) ornatus Pompecki, Pseudoasaphus tecticaudatus (Steinhardt), Mischynogorithes brachyrhachis Törnquist, Illaenus schroeteri schroeteri (Schlotheim), Nileus stigmatus Schrank, Pseudoasaphus aciculatus (Angelin), Cybellela sp., Remopleurides sp. Pseudomegalaspis patagiata (Törnquist), Cnemidopyge sp., Lonchodomas sp., Nieszkowskia cf. osmossaerensis Männil, Plectasaphus plicicostis (Törnquist), Telephina sp. aff. bicuspis (Angelin), Hibbertia cf. concavus (Thorslund), Lituites lituus (Modeer), Lituites perfectus (Wahlenberg), Cyclolituites lynceus Holm, Cyclolituites applanata Remelé, Clinoceras maskei (Dewitz), Trocholites

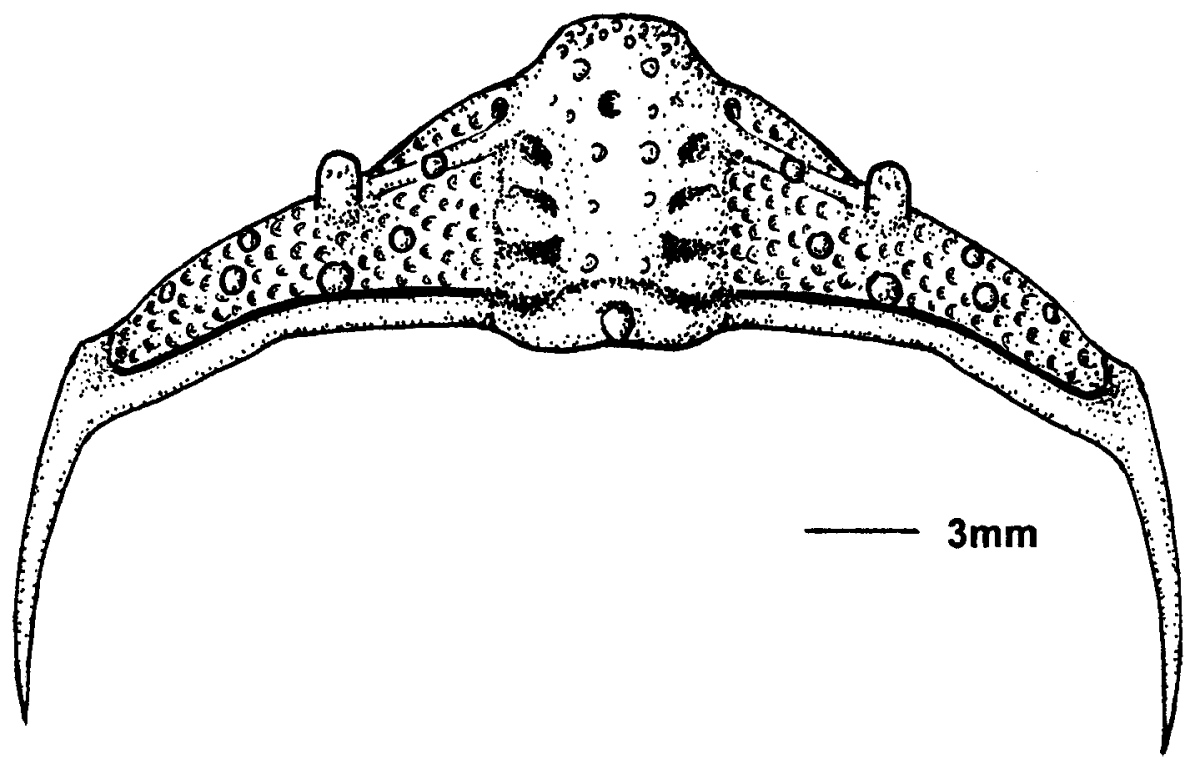

Abb. 2. Rekonstruktion von Atractocybeloides nebeni $\mathrm{n} . \mathrm{sp}$.

Tafel 1. 1-4. Atractocybeloides nebeni $\mathrm{n}$. sp. aus der Lasnamägi-Stufe $\mathrm{C}_{\mathrm{lb} \gamma}$, Folkeslundakalk, oberer grauer Orthocerenkalk, Mittl. Ordovizium, Cranidium, MB.T. 4492.1, Röbel (Müritz), Mecklenburg-Vorpommern. 1. Dorsal $\times 3$; 2 lateral $\times 3$; 3. frontal-dorsal $\times 3 ;$ 4. frontal $\times$ 3. 5. Atractocybeloides oepiki n. sp. aus der Kukruse-Stufe $C_{\text {II } \alpha}$ Kuckersit, Ober-Ordovizium, Cranidium, MB.T. 1693.2, Kohtla, Estland, dorsal $\times$ 7,5. 6-13. Atractocybeloides berneri Krueger, 1991 aus dem Backsteinkalk der Idavere- und Johvi-Stufe $\mathrm{C}_{\mathrm{III} \beta}-\mathrm{D}_{\mathrm{I}}$, Ober-Ordovizium; 6-8. Cranidium, MB.T. 4493.1, Wustrow, Fischland, MecklenburgVorpommern; 6 dorsal $\times 5$; 7. frontal-dorsal $\times 5$; 8. lateral-dorsal $\times 5$, coll. Berner; 9-11. Cranidium MB.T. 4494.1, Schlabendorf-Süd, Lausitz; 9. dorsal $\times 6$; 10. lateral-dorsal $\times 6$; 11. frontal-dorsal $\times 6$, coll. Krueger; 12. Pygidium, RGM 212508a, Uelsen, Niedersachsen, dorsal $\times 12 ; 13$. Pygidium, RGM 212504a, Uelsen, Niedersachsen, dorsal $\times 12 ; 12$ und 13. coll. Rhebergen, Emmen. 


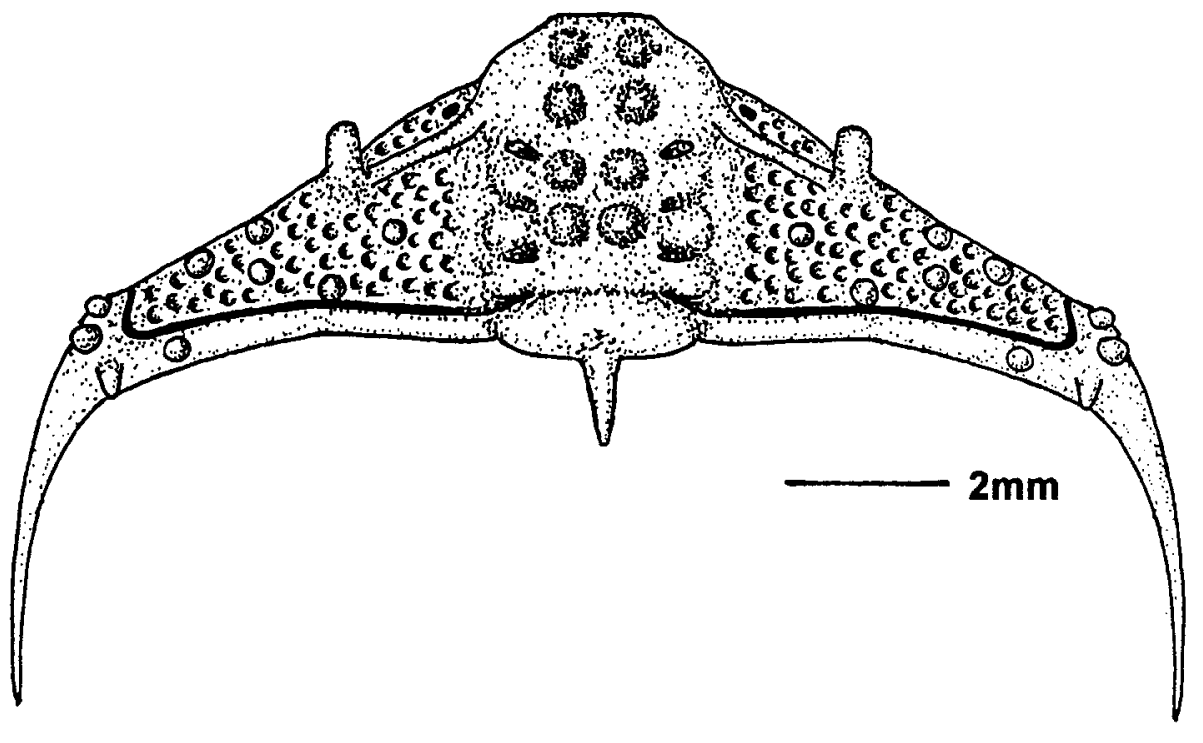

Abb. 3 Rekonstruktion von Atractocybeloides oepiki n. sp. sp., Raphistoma obvallatum (Wahlenberg), Clathrospira elliptica (Hisinger), Lesueurilla declivis (Remelé), Hyolithes acutus Eichwald. Diese Fauna ist typisch für den oberen Teil der Lasnamägi-Stufe $\mathrm{C}_{\mathrm{Ib} \gamma}$, dem Folkeslundakalk.

\section{Atractocybeloides oepiki n. sp.}

Tafel 1: 5, Abb. 3

Holoty pus: 1 Cranidium, MB.T.1693.2.

Locus typicus: Brandschieferbruch Kohtla, Estland. Stratum typicum: Kukruse-Stufe $\mathrm{C}_{\mathrm{II} \alpha}$, mittleres Viru. Derivatio nominis: Nach dem Miterforscher des Brandschiefers (Kuckersit) in Estland: A. Öpik.

Material: 1 Cranidium

Dieses kleine, zur Hälfte erhaltene Cranidium von $A$. oepiki n. sp. wurde bei Nachpräparationsarbeiten an Atractopyge rex-Material um 1970 gefunden. Es wurde von Öpik 1927 in Kuckers gesammelt und lag bis vor kurzem unerkannt in der Sammlung des Museums für Naturkunde.

Maße (in mm):

Glabella, größte Breite bei L1 $\approx 2,5$

Glabella, kleinste Breite Frontallobus $\approx 2,5$

Glabella + Occipitalring, Länge $\quad 3,4$

Occipitalring, Breite $\approx 2,8$

Cranidium, Breite $\quad \approx 10,4$

Diagnose: Frontallobusvorderkante in der Mitte gerade, an den Seiten abgeschrägt. Gla- bella mit acht paarig angelegten, sehr großen kegelförmigen Tuberkeln besetzt. Glabellarfurchen leicht nach vorn und nach außen gerichtet. Occipitalring median mit Stachel, breiten Festwangen und kräftigem Wangenstachel.

Beschreibung: Cranidium flach gewölbt; stärker erhöht nur Glabella und Occipitalring. Glabella vom gut entwickelten Occipitalring durch gerade, mäßig tiefe Furche getrennt. Occipitalring trägt median kräftigen, nach hinten gerichteten Stachel. L1-Loben gut entwickelt und rundlich, zum Occipitalring durch tiefe Lateralgruben abgegrenzt. Zu den L2-Loben durch tiefe, dreieckige Lateralfurchen abgesetzt. L2-Loben nicht so stark ausgebildet und wie die kräftigen L3Loben leicht nach vorn gerichtet. Durch mäßig tiefe Lateralfurchen untereinander abgegrenzt. Frontallobus vorn gerade, an den Seiten abgeschrägt und von den gut ausgebildeten Fossulagruben, die ihn von den vorderen inneren Festwangen absetzen, getrennt. Augenleisten verlaufen vom Frontallobus schräg nach hinten, um in den Augenstielen zu enden. (Augen nicht vorhanden, aber vermutlich wie alle anderen dieser Gattung gestielte Augen besitzend). Festwangen breit und zur Wangelstachelbasis relativ schmal werdend. Hinterrand bis in Höhe der Augen fast gerade verlaufend, um dann aber deutlich nach hinten zu knicken, rund und zur Wangenstachelbasis breit werdend, um in den kräftigen, im

Tafel 2. 1-7. Atractocybeloides berneri Krueger, 1991 aus dem Backsteinkalk der Idavere und Johvi-Stufe $\mathrm{C}_{\mathrm{III} \beta}-\mathrm{D}_{\mathrm{I}}$, OberOrdovizium, Slg. Rhebergen, Emmen. 1, 3 und 5. Cephalon mit zwei Thoraxsegmenten, RGM 212509, Uelsen, Niedersachsen; 1. dorsal $\times 6$; 3. frontal-dorsal $\times 6$; 5. lateral-dorsal $\times 6$; 2 . Thorax aus zwölf Segmenten, RGM 212501a, Uelsen, Niedersachsen, dorsal $\times 5,6 ; 4$. Cranidium, RGM 212502a, Uelsen, Niedersachsen, lateral-dorsal $\times 6,8 ; 6-7$. Pygidium, RGM 212503a, Uelsen, Niedersachsen, 6, dorsal $\times 8,3 ; 7$, lateral $\times 8,3$, coll. Rhebergen, Emmen. 

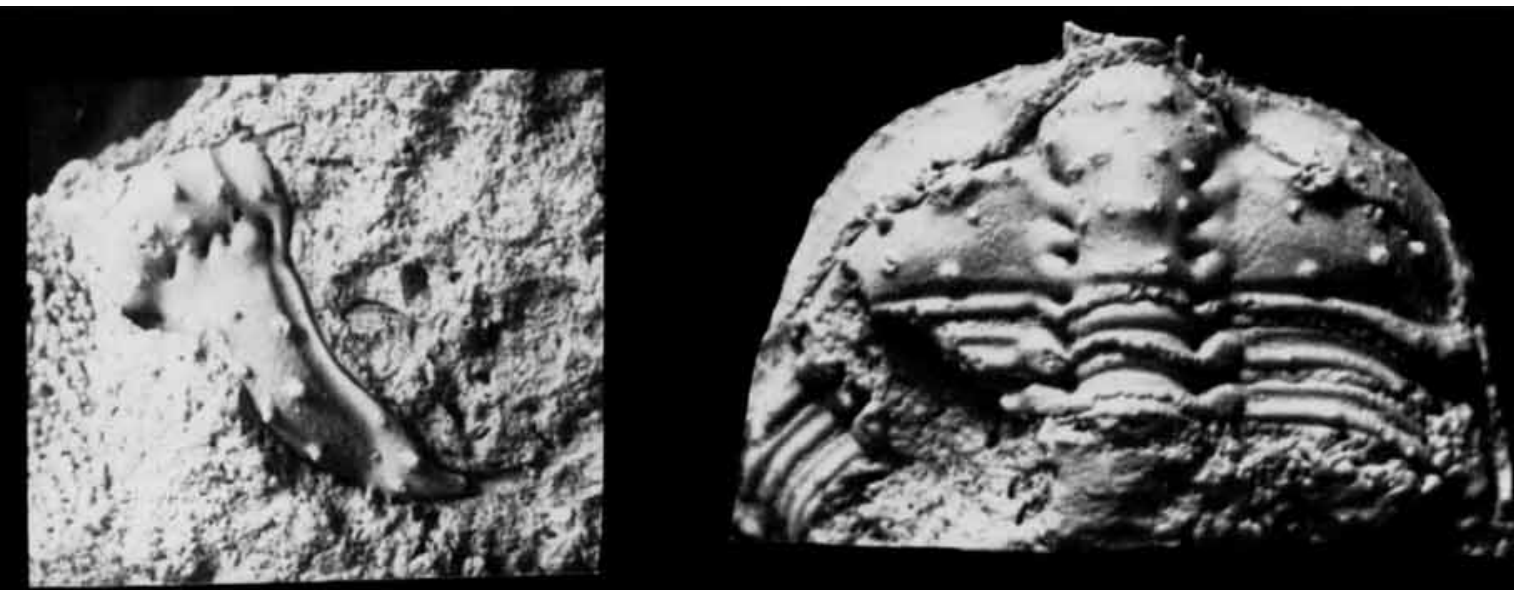

4

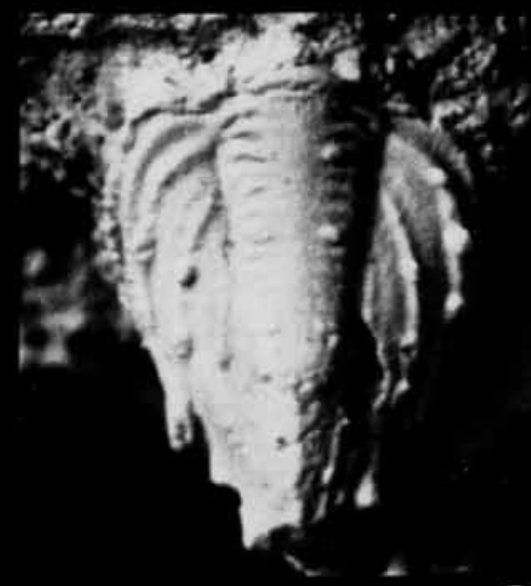

6
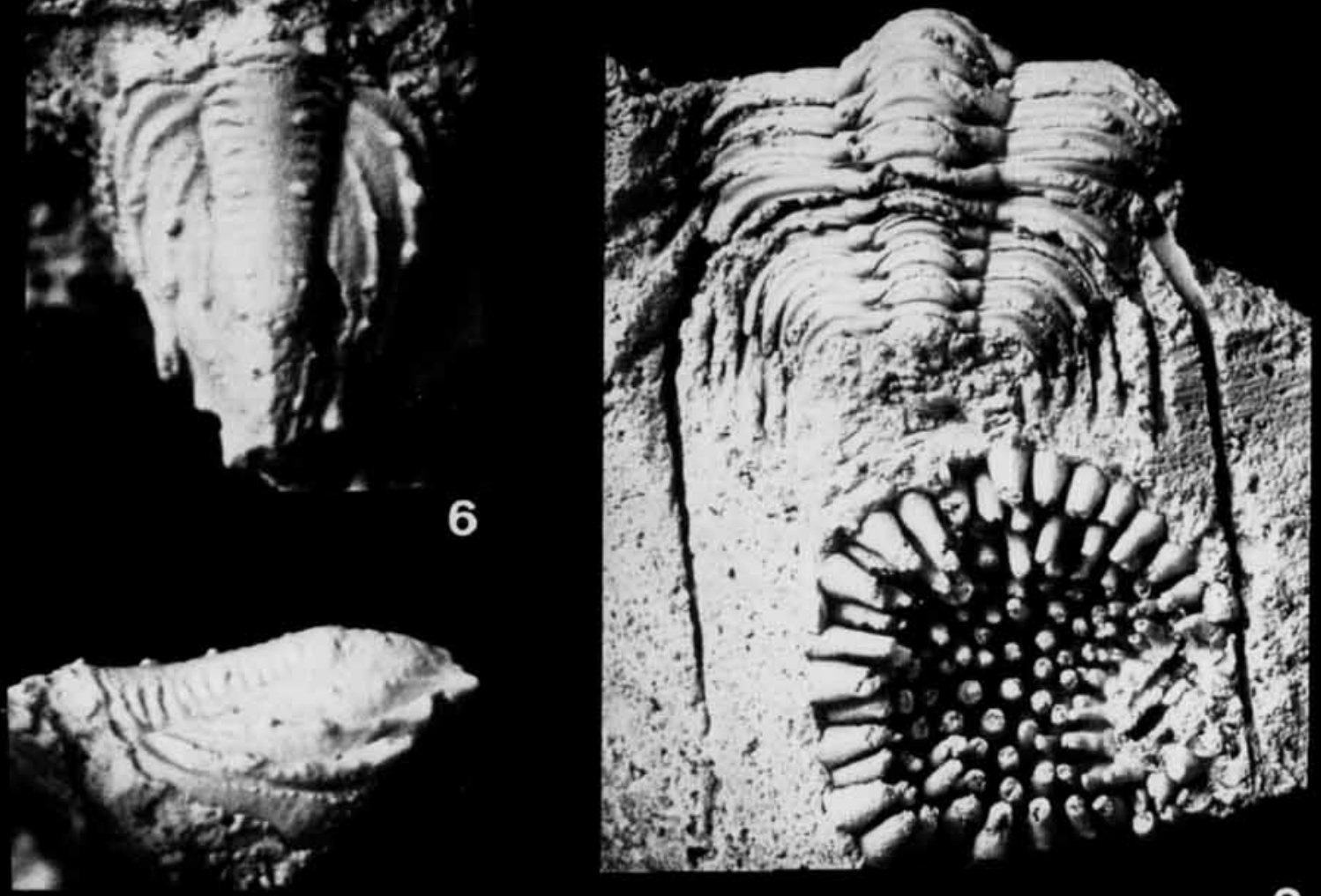

7
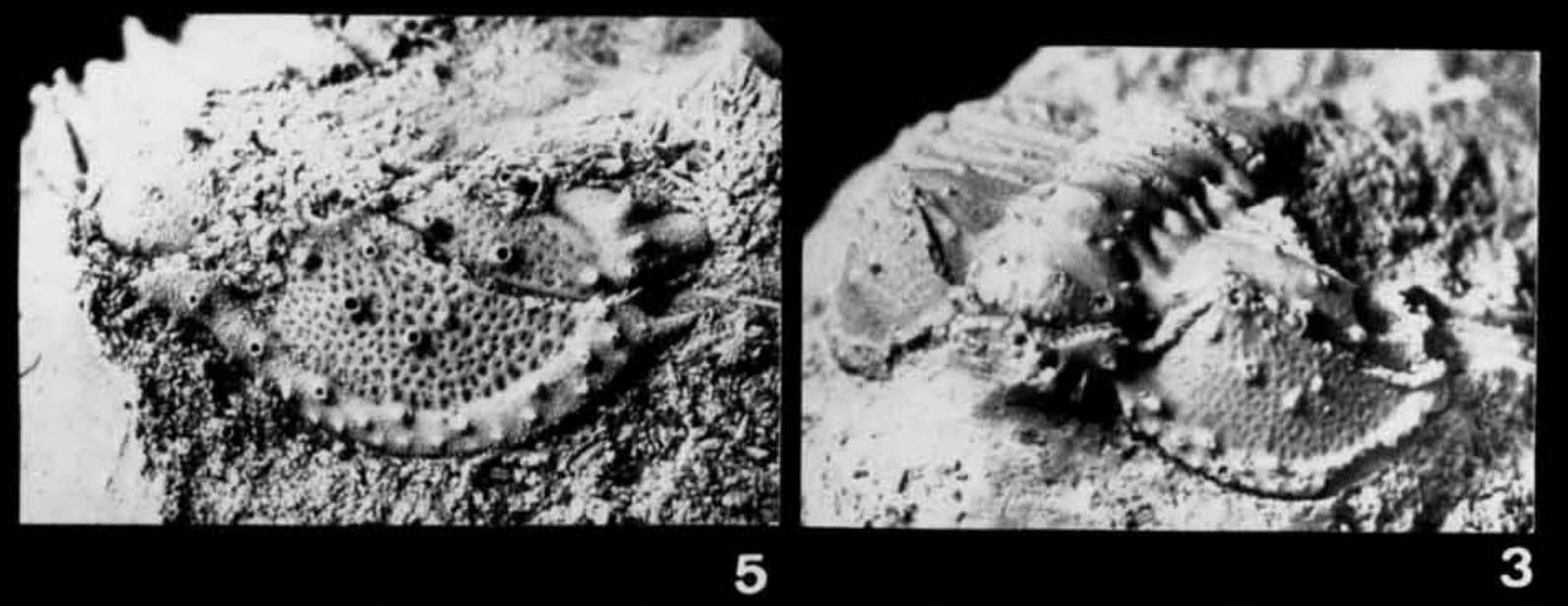
Querschnitt runden Stachel überzugehen, der nach hinten und leicht nach oben gerichtet ist. Gesichtsnaht fast gerade verlaufend, vom Frontallobus aus aber schräg über die Augen nach hinten und außen. Sehr große, paarig angeordnete, kegelförmige Tuberkeln, die die Glabella bedecken, vier bei L1-L3 liegend und die vier größten auf dem Frontallobus. Torulartuberkel auf Festwangen schwach ausgeprägt, PostocularTuberkel zwischen den Augen und der Wangenstachelbasis als großer Tuberkel angelegt. Tuberkeln auf dem äußeren Ende des Hinterrandes und der Wangenstachelbasis sehr groß und dornenartig. Schalenoberfläche auf den Festwangen mit flachen Grübchen versehen (Abb. 3).

Unterschiede: $A$. oepiki n. sp. unterscheidet sich von der älteren Art $A$. nebeni durch die starke Tuberkulierung auf der Glabella, dem mit großen Tuberkeln versehenen Hinterrand und die Wangenstachelbasis, weiterhin durch einen starken, dornartigen, nach hinten gerichteten Stachel auf dem Occipitalring. Festwangenaußenteile stärker nach hinten gerichtet.

Vorkommen: Die Fauna, in welcher Atractocybeloides oepiki $\mathrm{n}$. sp. vorkommt, ist sehr reichhaltig und repräsentiert den unteren Teil der Kukruse-Stufe $\mathrm{C}_{\mathrm{II} \alpha}$ mit dem typischen Brandschiefer von Kohtla und Maidla in Estland. Mit ihr haben sich Fr. Schmidt (1881), Öpik (1925, 1926, 1937) und Roomusoks (1970) eingehend beschäftigt.

\section{Atractocybeloides berneri Krueger, 1991}

Tafel 1: 6-13, Tafel 2:1-7, Tafel 3: 1-2, Abb. 4

Material: 1 Cranidium, Holotyp, Slg. H.-H. Krueger Nr. 1559.1, jetzt Slg. I.f.P., MfN Berlin MB.T. 4493.1 und 1 Cranidiumfragment $\mathrm{Nr}$. S.70.1, jetzt Slg. I.f.P., MfN Berlin, MB.T. 4494.1, 1 Panzer ohne Pygidium RGM 212501a, 1 Cephalon mit 2 Thoraxsegmenten RGM 212509a, Cephalon mit 11 Thoraxsegmenten RGM 212510a, 1 Cranidium RGM 212502a, 3 Pygidien RGM 212503a, 212504a, 212508a, 2 Freiwangen 212505a, 212507a, zahlreiche Freiwangen und Pygidien.
Maße (in mm):

\begin{tabular}{|c|c|c|c|c|c|}
\hline & $\begin{array}{l}\text { Holo- } \\
\text { typus } \\
\text { MB.T. } \\
4493.1\end{array}$ & $\begin{array}{l}\text { RGM } \\
212509 a\end{array}$ & $\begin{array}{l}\text { RGM } \\
212501 \mathrm{a}\end{array}$ & $\begin{array}{l}\text { RGM } \\
212510 \mathrm{a}\end{array}$ & $\begin{array}{l}\text { MB.T. } \\
4494.1\end{array}$ \\
\hline $\begin{array}{l}\text { Glabella, } \\
\text { größte Breite L1 }\end{array}$ & 3,5 & 2,5 & 3,0 & 2,0 & 2,1 \\
\hline $\begin{array}{l}\text { Glabella, } \\
\text { kleinste Breite } \\
\text { Frontallobus }\end{array}$ & 3,0 & 2,3 & 2,7 & 1,9 & 2,0 \\
\hline $\begin{array}{l}\text { Glabella + } \\
\text { Occipitalring, } \\
\text { Länge }\end{array}$ & 5,0 & 4,0 & 4,3 & 3,2 & 3,1 \\
\hline $\begin{array}{l}\text { Occipitalring, } \\
\text { Breite }\end{array}$ & 3,8 & 2,5 & 2,8 & 2,1 & 2,2 \\
\hline $\begin{array}{l}\text { Cranidium, } \\
\text { Breite }\end{array}$ & 10,8 & 10,6 & 10,5 & 9,4 & - \\
\hline
\end{tabular}

Emendierte Diagnose: Cranidium flach gewölbt, nur an den Seiten steiler, Frontallobusvorderkante in der Mitte gerade, zu den Seiten abgeschrägt, Präglabellarfeld nicht ausgebildet. Frontallobus mit vier großen und mehreren kleineren Tuberkeln bedeckt. Mediangrube vorhanden. Übrige Glabella mit vier paarig angeordneten Tuberkeln versehen. Glabellarfurchen leicht nach vorn und außen gerichtet. Occipitalring median mit einem Stachel, Festwangen breit, Augen gestielt, Freiwangen mit gerundetem Außenrand, Wangenstachel lang. Thorax aus zwölf Segmenten, sechste Thoraxpleuren zu langen Stacheln ausgezogen. Pygidium aus vier Rippen, schmal.

Beschreibung: Cephalon flach gewölbt, nur zu den Seiten in Richtung der Wangenstacheln und Freiwangen steiler abfallend. Glabella gleichmäßig gebogen, höchster Punkt beim zweiten Tuberkelpaar, zwischen L2 und L3. Vom Occipitalring durch eine breite, flache Furche, die leicht in der Mitte bogenförmig nach vorn vorgezogen ist und an den Seiten in tiefen Gruben endet, getrennt. Occipitalring kräftig, gleichmäßig gebogen wie die Glabella, in der Mitte mit einem spitzen, runden Stachel versehen, der leicht nach oben und nach hinten bis zum zweiten Thoraxsegment reichen kann. Er wird von je einem kleinen Tuberkel flankiert. Erstes Laterallobenpaar kräftig, leicht gerundet und schwach nach vorn gerichtet, durch tiefe und breite Glabellarfurchen von dreieckiger Form von den zweiten Loben abgegrenzt. Zweite Lateralloben leicht nach vorn verlaufend, im Mediankörper etwas schmaler und tiefer ansetzend, $\mathrm{zu}$ den Festwangen hin breiter werdend. Dritte Lateralloben ähnlich kräftig wie die ersten Loben entwickelt und leicht nach vorn gerichtet. Von den 

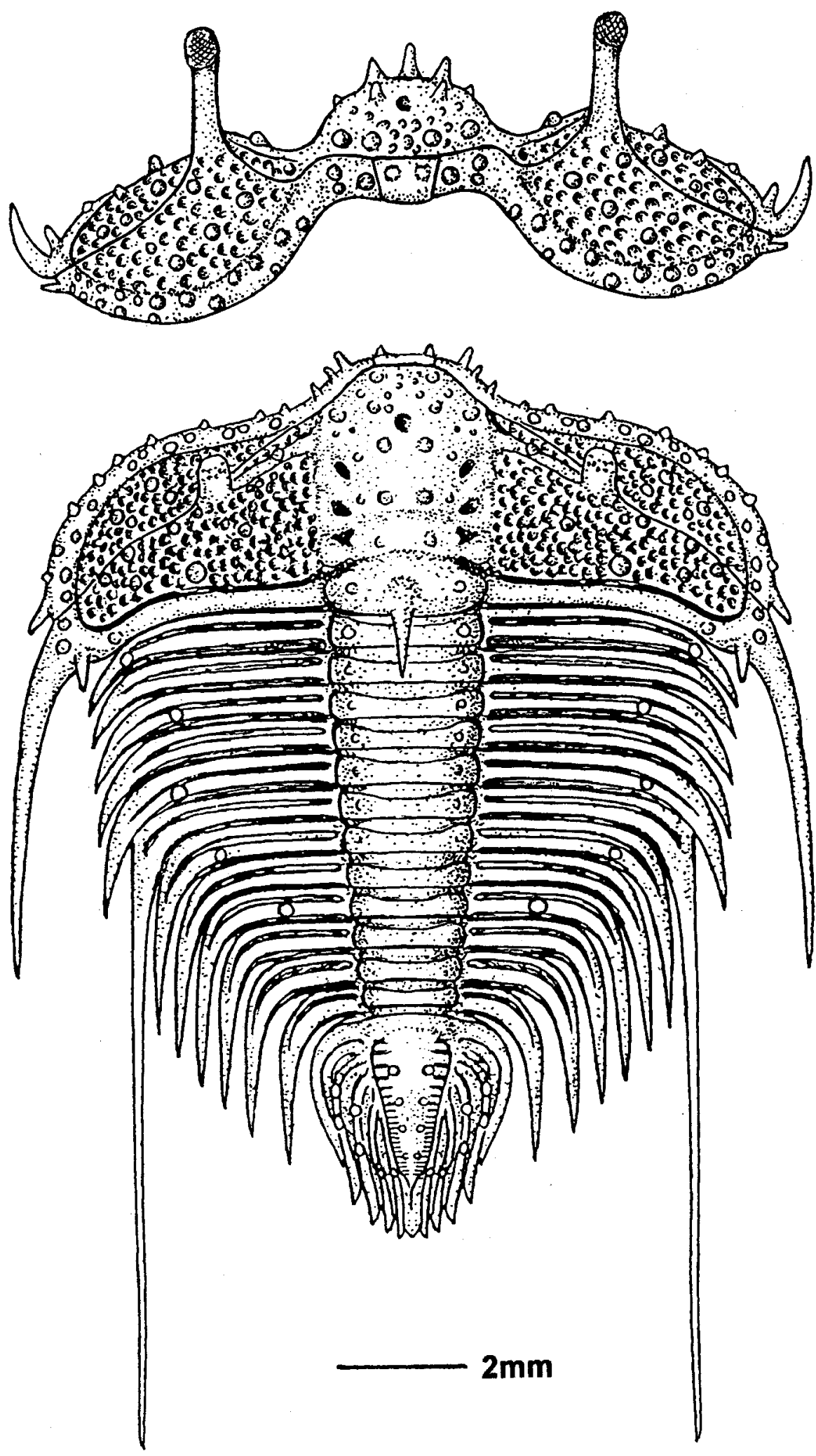

Abb. 4. Rekonstruktion von Atractocybeloides berneri Krueger, 1991.

zweiten Loben durch tiefe, nach außen breiter werdende Glabellarfurchen abgesetzt. Dritte Loben nach vorn durch Glabellafurchen, die tief, breit und oval sind, vom Frontallobus getrennt. Die einzelnen Lobenpaare im Übergang zu den Festwangen miteinander verwachsen und von den Festwangen durch die kaum wahrnehmbaren Dorsalfurchen abgesetzt. Frontallobus ungefähr ein Drittel breiter als Mediankörper der Glabella. Frontallobus-Vorderkante in der Mitte gerade und zu den Seiten nach hinten abgeschrägt. Diese Schräge läuft in vorderer Begrenzung der Dorsalfurchen, wo auch die gut entwickelten Fossulagruben liegen, aus. Präglabellarfeld nicht ausgebildet. Frontallobus mit sechs großen Tuberkeln besetzt, wovon sich je einer in Höhe des 
hinteren Endes der Schräge befindet, sowie ungefähr sechzehn kleinen Tuberkeln, die vor allem im vorderen Teil der Glabella postiert sind. Mediangrube sehr flach im hinteren Teil des Frontallobus. Mediankörper mit drei paarig angeordneten Tuberkeln versehen, wovon die bei L1 etwas kleiner sind. Tuberkeln bei L2 und zwischen L3 und dem Frontallobus deutlich größer und von je einem kleinen Tuberkel außen flankiert (Tafel 1: 9-11 und Tafel 2: 1).

Festwangen schwach gebläht, mäßig hoch, recht breit, nach außen zum Wangenstachel steil abfallend. Augenstiele schlank, ungefähr drei Viertel der Länge der Glabella erreichend. Augenstiele durch eine Leiste, die einen Tuberkel trägt, mit dem Frontallobus verbunden. Zwischen den Augenstielen und der Wangenstachelbasis fünf große Tuberkeln, wovon drei an der Gesichtsnaht liegen. Postoculartuberkel dicht an der Hinterrandfurche, die fast gerade nach außen verläuft und erst kurz vor der Wangenstachelbasis leicht nach hinten biegt, um dann im scharfen Bogen nach vorn zu biegen. Hinterrand leicht gerundet, gerade nach außen gerichtet, erst kurz vor der Wangenstachelbasis breiter werdend und in weichem Bogen in den Wangenstachel übergehend. Wangenstachel in leichtem Bogen nach hinten und oben gerichtet, rund, ungefähr eineinhalbmal so lang wie die Glabella. Wangenstachelbasis mit drei großen Tuberkeln besetzt, wovon der hintere als kurzer Stachel ausgebildet ist. Ein kleiner Tuberkel kann sich kurz vor diesem auf dem Hinterrand befinden (Tafel 1: 6, 8 und Tafel 2: 1,4-5).

Gesichtsnaht leicht schräg nach hinten verlaufend, im äußeren Teil nach außen abgeschrägt. Glabellaoberfläche, Occipitalring und Wangenstachel fein granuliert, Festwangen dicht mit flachen Grübchen bedeckt.

Freiwange: Freiwangenfeld dreieckig und vom Außenrand durch eine flache Furche abgesetzt, die nach vorn tiefer wird und das kleine Pseudoglabellarfeld vom Freiwangenfeld trennt. Außen- rand nach außen und unten gleichmäßig gebogen. Er ist leicht abgeflacht und endet im Pseudoglabellarfeld, das von fast rechteckiger Gestalt ist, wobei die obere Begrenzung leicht nach vorn abgeschrägt ist. Schnauzenschild (Scutum rostrale) klein, trapezförmig ausgebildet und beide Freiwangen mit dem Vorderrand des Frontallobus verbindend. Freiwangenaußenrand mit großen alternierenden Tuberkeln, dazwischen kleine Tuberkeln. Auf dem Pseudoglabellarfeld drei große Tuberkeln, von denen der untere, innere stachelförmig ist. Vor der Gesichtsnaht ist der Außenrand mit einem Stachel versehen. Freiwangenfeld mit fünf großen Tuberkeln bedeckt, wovon zwei parallel zur Gesichtsnaht liegen und drei etwas tiefer in Reihe angeordnet sind. Augenstiele schlank und ihre Länge erreicht ungefähr die Höhe des Freiwangenfeldes. Die Sehfläche ist sehr klein. Freiwangenfeld dicht mit flachen Grübchen bedeckt.

Hypostom: Von allen hier vorgestellten Arten ist kein Hypostom bekannt. Es dürfte vom Typ Cybeloides virgeniensis virgeniensis sein (siehe Evitt \& Tripp 1977: Tafel 17: 17-19).

Thor ax: Thorax setzt sich aus zwölf Segmenten zusammen, die außen an den Pleurenenden in nach hinten und leicht nach außen gerichtete Spitzen ausgezogen sind, sechstes Segment in einen langen, schlanken Stachel ausgezogen, der weit über das Pygidium reicht. Übrige Pleurenenden bis zum Pygidium als gleichmäßige, mittellange Stacheln ausgebildet. Hinteres Pleurenband größer, kräftiger. Mittelachse erreicht ungefähr ein Drittel der Thoraxbreite und ist ungefähr ein Drittel höher als die Pleuren. Vereinzelt kann auf dem hinteren Pleurenband im Außenteil ein spitzer Tuberkel postiert sein. Auch sind auf den ersten Axialringen paarige, kleine Tuberkeln vorhanden.

Pygidium: Pygidium von triangulärem Umriss, ungefähr ein Drittel länger als breit. Rhachisbreite beträgt ein Drittel der Pygidiumbreite, Rhachis flach gewölbt und leicht durchgebogen.

Tafel 3. 1-2. Atractocybeloides berneri Krueger, 1991 aus dem Backsteinkalk der Idavere und Johvi-Stufe $C_{\Pi 1} \beta-D_{I}$, OberOrdovizium. 1. Linke Freiwange, RGM 212507a, Uelsen, Niedersachsen, lateral $\times 11,5 ; 2$. rechte Freiwange mit Augenstiel, RGM 212505, Uelsen, Niedersachsen, lateral $\times 10$, coll. Rhebergen, Emmen. 3-6. Atractocybeloides vonhachti Krueger, 1991 aus verkieselten Kalken und Sandsteinen des unteren Teils der Keila-Stufe $D_{\mathrm{Il} a}$, Ober-Ordovizium. 3. Cranidium, MB.T. 4495.1, Schlabendorf Süd, Lausitz, dorsal $\times 10$, coll. Krueger; 4. Linker Wangenstachel, MB.T. 4496.1, Schlabendorf-Süd, Lausitz, lateral-dorsal $\times 7$, coll. Krueger; 5-6. Cranidium, RGM 212506a, Uelsen, Niedersachsen; 5. dorsal $\times 5$; 6. frontal-dorsal $\times 5$, coll. Rhebergen, Emmen. 7-9. Atractocybeloides gracilis (Nikolaisen, 1961) aus dem oberen Chasmops-Kalkstein $(4 \mathrm{~b} \delta)=$ Rakvere-Stufe E, Ober-Ordovizium. 7. Cranidium, P.M.O.5600, Terneholmen, Asker bei Oslo, Norwegen, dorsal $\times 3$; 8. Cranidium, P.M.O.6531, Terneholmen, Asker bei Oslo, Norwegen, dorsal $\times 3$; 9. Pygidium, P.M.O.9095, Terneholmen, Asker bei Oslo, Norwegen, dorsal $\times 6$, coll. Kiaer. 7-9. aus Nikolaisen (1961). 

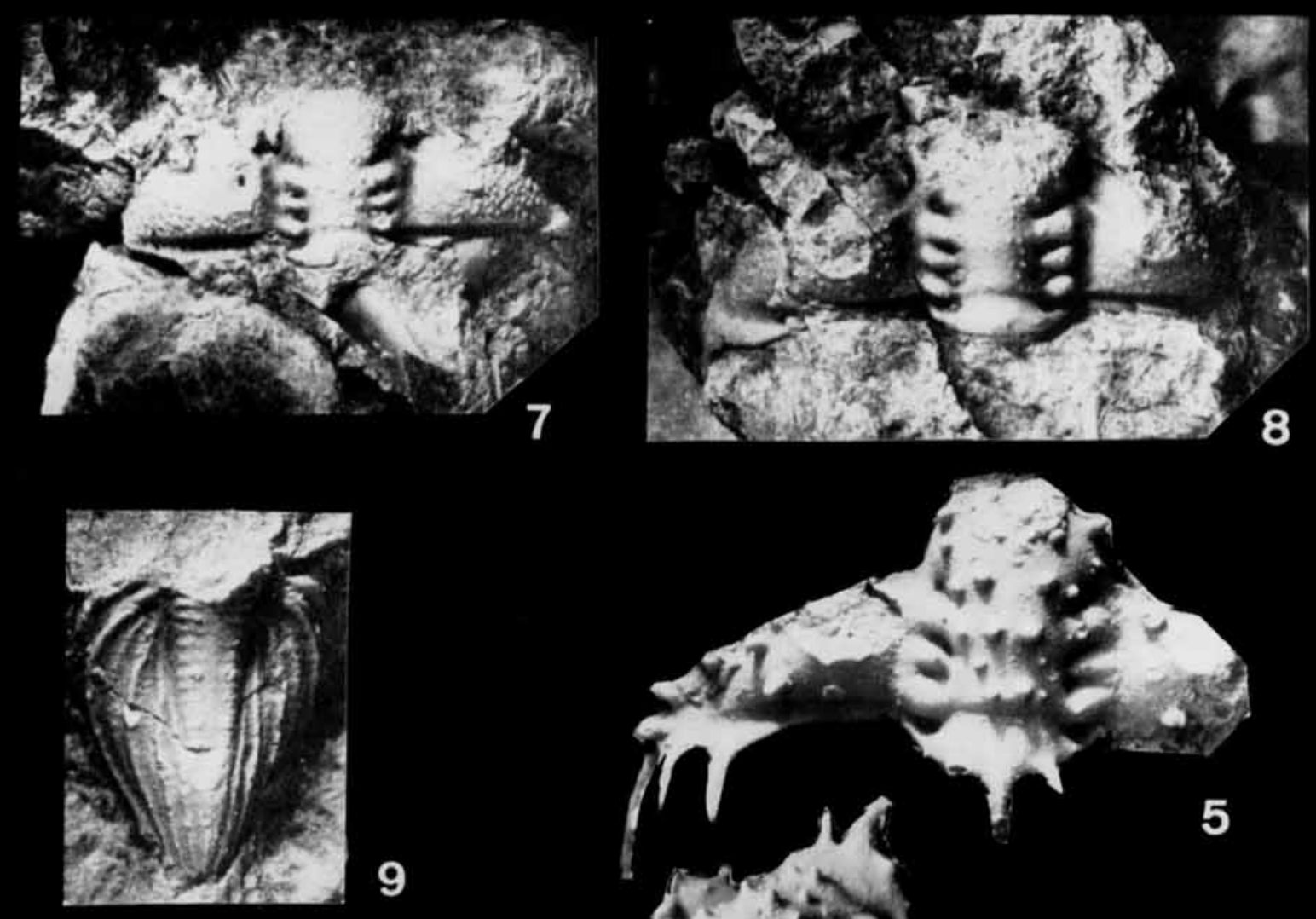

9

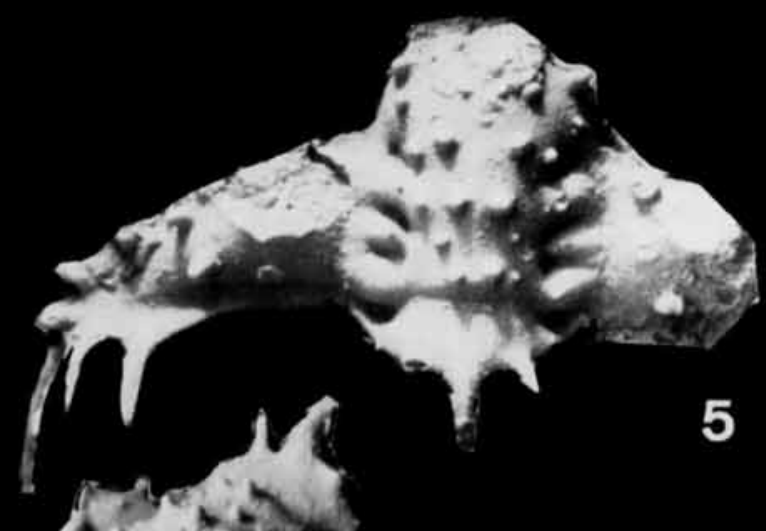

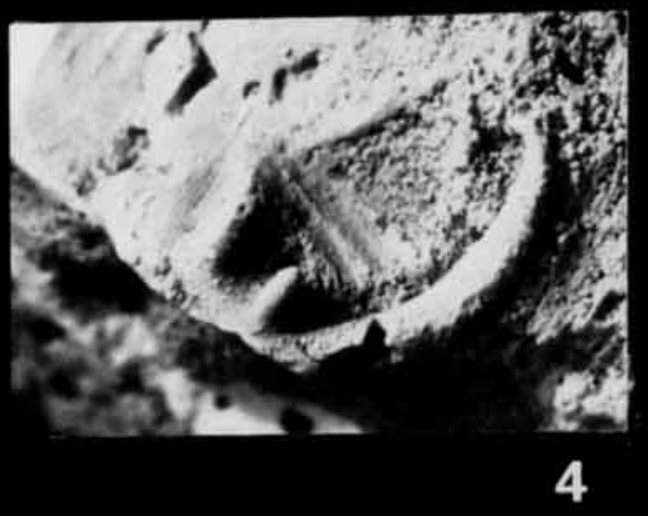

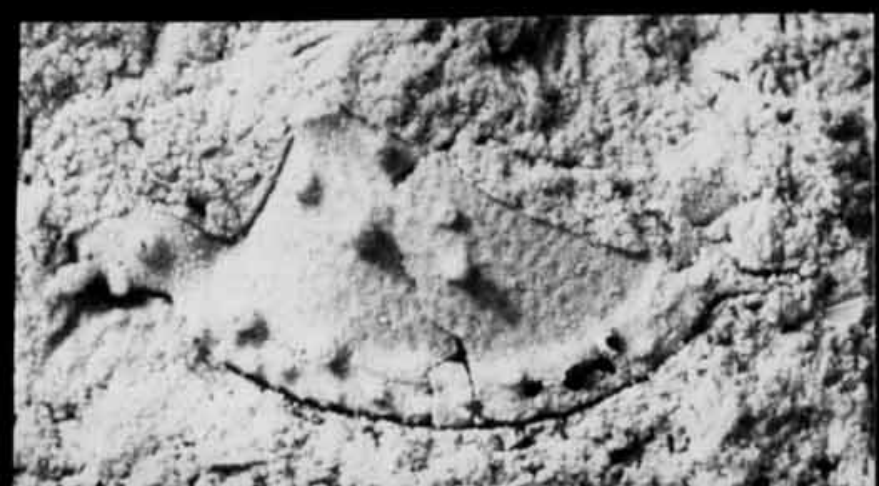

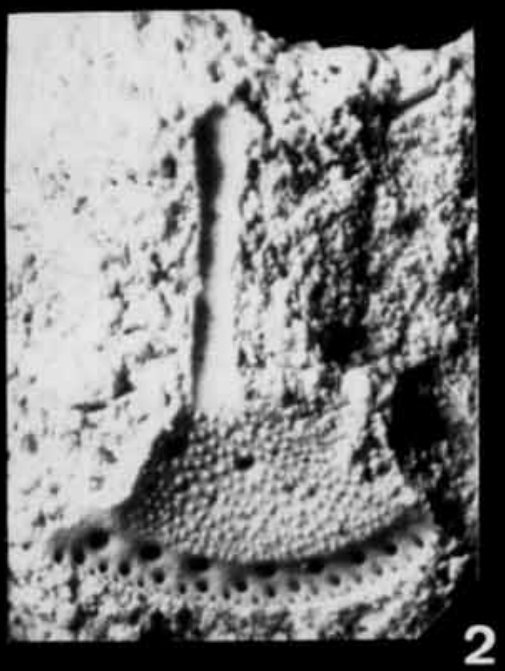


Ihre Länge erreicht ungefähr zwei Drittel der Pygidiumlänge. Rhachis setzt sich als Grat zum Pygidiumende fort. Vier paarig angelegte Tuberkeln sind an den Seiten der Rhachis postiert. Rhachisringe (sechszehn bis achtzehn) nur an den Seiten der Rhachis angelegt. Vier rippenartige Pleuren bedecken die Pygidiumseitenteile, wovon die beiden ersten im Bogen nach außen und hinten gerichtet sind und die beiden inneren parallel mit der Rhachis verlaufen. Rippenpleuren können mit ein bis zwei großen Tuberkeln besetzt sein. Hinten enden die Pleuren in feinen Spitzen, wobei die Spitzen der beiden ersten Rippenpleuren leicht nach außen gerichtet sind. Der Pygidiumtyp ist der von Cybelloides und Atractopyge (Tafel 1: 12-13, Tafel 2: 6-7).

Unterschiede: Atractocybeloides berneri unterscheidet sich von $A$. nebeni $\mathrm{n}$. sp. durch die deutliche und reichere Tuberkulierung auf der Glabella und die Vergrößerung der Tuberkeln auf dem Hinterrand und der Wangenstachelbasis. A. berneri nimmt zwischen $A$. nebeni $\mathrm{n}$. sp. und $A$. vonhachti eine Zwischenstellung ein, da $A$. vonhachti stärker tuberkuliert und mit Stacheln versehen ist.

Vorkommen: Atractocybeloides berneri Krueger kommt zusammen vor mit Asaphus (Postasaphus) cf jewenensis F. Schmidt, Bolbochasmops emarginata (F. Schmidt), Chasmops marginata (F. Schmidt), Oculichasmops mutica (F. Schmidt), Conolichas monticulosus (Öpik), Cybellela cf. dentata (Esmark), Illaenus jewensis Holm, Hemisphaerocoryphe pseudohemicranium (Nieszkowski), Niezkowskia sp. Clitambonites schmidti epiconus Öpik, Cyrtonotella kuckersiana frechi (Wysogorski), Oepikia anijana anijana (Öpik), Leptaena sp., Platystrophia chama (Eichwald), Platystrophia lynx lynx (Eichwald), Porambonites baueri Noetling, Pseudolingula sp., Sowerbyella (S.) plana Roomusoks, Lesueurilla sp., Salpingostoma compressum Eichwald, Subulites (S.) amphora (Eichwald), Rectanguloceras sp., Mastopora concava Eichwald und Diplotrypa sp. Diese Fauna repräsentiert die obere Idavere-Stufe $\mathrm{C}_{\mathrm{III} \beta}$ und Teile der Johvi-Stufe $\mathrm{D}_{\mathbf{I}}$.

\section{Atractocybeloides vonhachti Krueger, 1991}

Tafel 3: 3-6, Abb. 5

Material: 1 Cranidium-Abdruck, Holotypus, Nr. G113/1, Archiv für Geschiebekunde Hamburg, coll. U. v. Hacht; 1 Cranidium, MB.T. 4495.1, coll. Krueger; 1 Cranidium, RGM 212506a, Slg. Rhebergen und 1 Fragment vom linken Hinterrand mit Wangenstachel, MB.T.4496.1, coll. Krueger
$\mathrm{Maße}($ in $\mathrm{mm})$ :

\begin{tabular}{|c|c|c|c|}
\hline & $\begin{array}{l}\text { Holotyp } \\
\text { G } 113 / 1\end{array}$ & $\begin{array}{l}\text { MB.T. } \\
4495.1\end{array}$ & $\begin{array}{l}\text { RGM } \\
212506 a\end{array}$ \\
\hline $\begin{array}{l}\text { Glabella, } \\
\text { größte Breite L1 }\end{array}$ & 4,5 & 1,4 & 4,4 \\
\hline $\begin{array}{l}\text { Glabella, } \\
\text { kleinste Breite Frontallobus }\end{array}$ & 4,0 & 1,4 & 3,7 \\
\hline $\begin{array}{l}\text { Glabella + Occipitalring, } \\
\text { Länge }\end{array}$ & $\approx 6,2$ & 2,4 & 5,9 \\
\hline Occipitalring, Breite & 4,7 & 1,5 & 4,7 \\
\hline Cranidium, Breite & 20,5 & 8,0 & 20,0 \\
\hline
\end{tabular}

Emendierte Diagnose: Cranidium flach gewölbt, nur an den Seiten steiler abfallend, Frontallobusvorderkante gerade, an den Seiten abgeschrägt. Frontallobus mit Mediangrube und sechs paarig angeordneten großen Tuberkeln bedeckt, übrige Glabella mit vier paarig angeordneten großen Tuberkeln versehen. Occipitalring trägt drei Stachel, Lateralfurchen kräftig und tief, Festwangen mit Grübchen und sechs großen Tuberkeln auf jeder Wange. Augen gestielt, Hinterrand kurz vor dem Wangenstachel mit zwei großen langen Stacheln bestückt.

Beschreibung: Cranidium mittelkräftig gewölbt, nach außen zu den Wangenstachel steiler abfallend. Glabella gleichmäßig gewölbt, nur in Höhe der L3-Loben schräg nach vorn abgeflacht. Frontallobus vorn gerade begrenzt, an den Seiten abgeschrägt. Zwischen Frontallobus und Mittelteil der Glabella Gesichtsnaht schräg nach hinten verlaufend und in die Augenstiele übergehend. Am Knick zwischen Gerade und Schräge sitzt vorn an der Kante ein kleiner Tuberkel. Im Mittelteil des Frontallobus flache Mediangrube ausgebildet. Oberfläche des Frontallobus im hinteren Teil mit vier großen Tuberkeln und zwei vorn an der geraden Vorderkante, sowie bis zu zehn kleinen Tuberkeln im vorderen Medianbereich. An den Seiten des Frontallobus in die Augenleisten übergehend, schräg nach hinten zu den Augen verlaufend, von den L3-Loben durch kurze, mitteltiefe, schräg nach vorn und außen gerichtete Lateralfurchen getrennt. L3 kräftig, leicht nach vorn gerichtet, von den tiefer am Mediankörper der Glabella ansetzenden knotenförmigen L2-Loben durch tiefe mittelbreite Lateralfurchen, die leicht nach vorn stehen, abgesetzt. L1-Loben kräftig, von kugeliger Form und von L2-Loben durch dreieckige, tiefe Lateralfurchen abgesetzt. Zum Occipitalring L1-Loben außen von tiefen, ovalen Lateralfurchen getrennt. Occipitalring gut entwickelt, im Mittelteil 
breit, von der Glabella durch eine vorn in der Mitte leicht nach vorn gebogene, flache Furche abgesetzt. Im hinteren Medianbereich des Occipitalringes ein runder leicht nach oben und hinten gerichteter kräftiger Stachel, ungefähr bis zum zweiten Thoraxsegment reichend, an den Seiten von je einem kurzen Stachel flankiert. Seitenloben zu den Festwangen durch sehr flache, kaum wahrnehmbare Dorsalfurchen abgegrenzt. Mediankörper der Glabella zwischen L1 und $\mathrm{L} 2$ und bei $\mathrm{L} 3$ von großen, paarig angeordneten Tuberkeln besetzt, die außen und auf den Lateralloben vereinzelt mit kleinen Tuberkeln versehen sein können. Augenleisten sind mit einem sehr großen, stachelartigen Tuberkel versehen. Augenstiele kräftig und kürzer als bei A. berneri, aber auch unterhalb des Augendeckels mit einem Tuberkelkranz versehen.

Festwangen breit, flach gewölbt und nach auBen steiler abfallend. Verlauf der Gesichtsnaht schräg nach außen und hinten. Festwangen mit flachen Grübchen bedeckt. Fünf große, stachelartige Tuberkeln auf der Festwange, von denen zwei an der Gesichtsnaht liegen, dazu kommen Torular- und Postoculartuberkel. Hinterrand in weichem Bogen leicht nach hinten schwingend und dann in kräftige, runde, nach hinten und oben gerichtete Wangenstacheln übergehend. Wangenstachelbasis gut entwickelt, mit zwei großen, stachelartigen Tuberkeln besetzt. Hinterrand außen, kurz vor dem Wangenstachel mit zwei sehr großen, dornenartigen nach oben und hinten gerichteten Stacheln versehen (Tafel 3: 3-6; Abb. 5).

Bemerkungen: Atractocybeloides vonhachti wurde 1991 anhand von Resten zweier Cranidien und einem Fragment-Hinterrand mit Wangenstachel beschrieben. Ein weiterer Fund aus der Sammlung Rhebergen ermöglichte es, leichte Korrekturen an der damaligen Rekonstruktion des Cranidiums vorzunehmen. Anzunehmen ist,

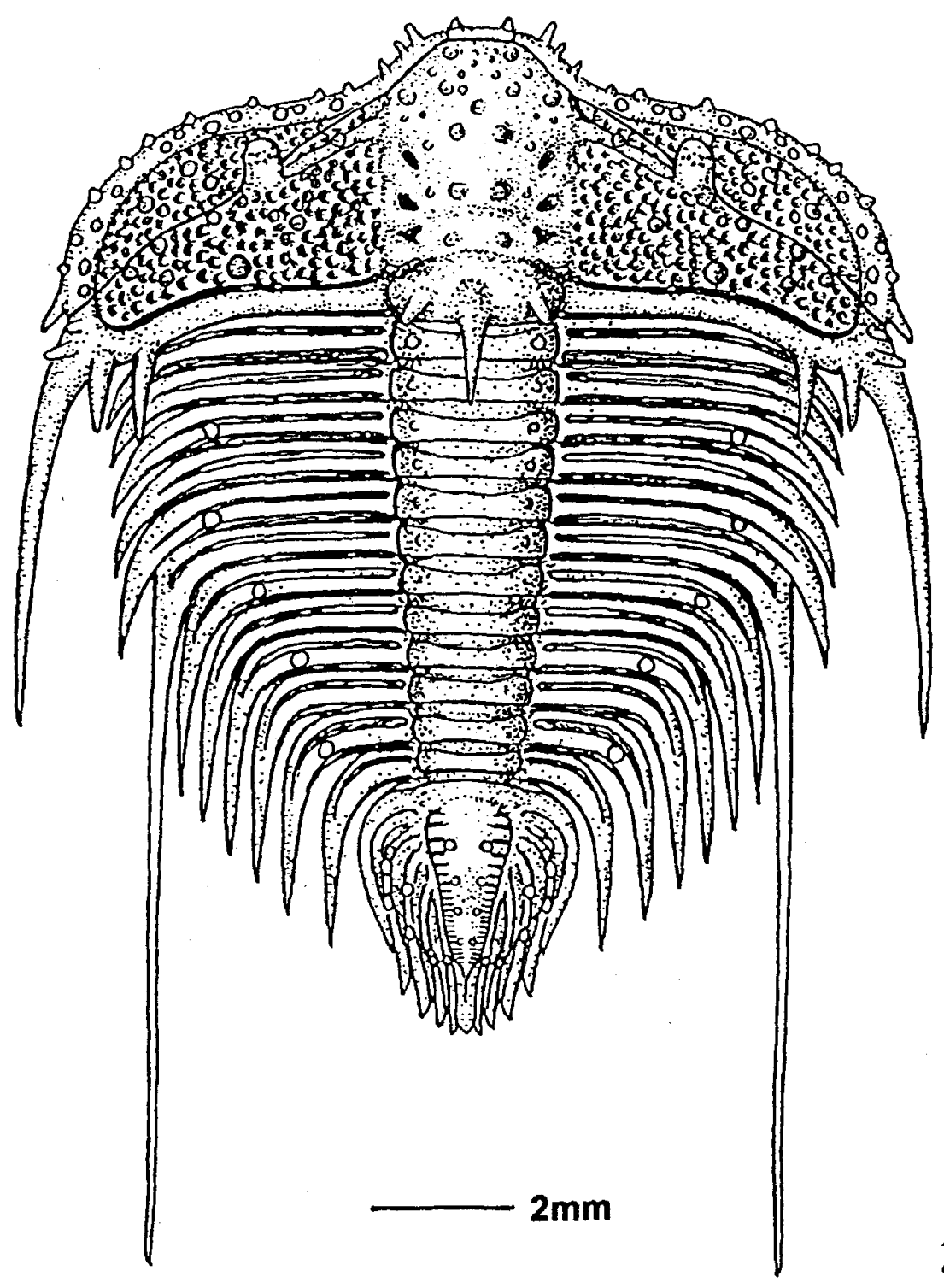

Abb. 5. Rekonstruktion von Atractocybeloides vonhachti Krueger, 1991. 
daß die vorderen Axialringe des Thoraxes kurze paarige Stachel trugen.

Unterschiede: Atractocybeloides vonhachti unterscheidet sich von den älteren Arten, $A$. nebeni n. sp., A. oepiki, A. berneri und der jüngeren Art aus dem Oslogebiet, durch seine extreme Bestachelung und seine großen Tuberkeln (Tafel 3: Figs 4-6).

Vorkommen: Die Panzerreste bei Atractocybeloides vonhachti Krueger sind nur als Cranidien bekannt. Das Material stammt aus Geröllen und Geschieben, die im Mittelmiozän als Verkieselungen in die Deckschichten über dem 2. Lausitzer Flöz abgelagert wurden, weiterhin von Sylt, aus Geröllen an der Grenze Pliozän/Pleistozän, sowie aus den Wilsumer Bergen, wo das Material im Menap abgelagert wurde. Die Begleitfauna setzt sich zusammen aus den Trilobiten Achatella cf. kegelensis (F. Schmidt), Atractopyge cf. errans Öpik, Cybellela sp., Toxochasmops (Schmidtops) sp., Chasmops marginatus (F. Schmidt), Asaphus (Neoasaphus) sp., Pharostomina sp., Nieszkowskia sp. sowie den Brachiopoden Leptaena sp., Whitfieldella? und Orthis sp. Diese Fauna repräsentiert den tiefen Teil der Keila-Stufe $\mathrm{D}_{\text {II } \alpha}$. Das Material liegt in verkieseltem Sandstein, auch ist Pharostomina für Baltoskandia fremd. In normalen Kalkgeschieben ist Atractocybeloides vonhachti noch nicht beobachtet worden.

\section{Atractocybeloides gracilis (Nikolaisen, 1961)}

Tafel 3: 7-9, Abb. 6

1961 Atractopyge gracilis n. sp. - Nikolaisen, S. 302-304, Tafel 4: Figs 5-9.

Material: 9 Cranidien, 2 Freiwangen und 11 Pygidien. Die Nummern zu diesen Stücken sind bei Nikolaisen (1961: 302) aufgeführt.

Emendierte Diagnose: Glabella flach gewölbt, Festwangen mehr gewölbt, zu den Wangenstacheln steiler abfallend. Augenstiele zierlich, Dorsalfurchen etwas deutlicher als bei den älteren Arten. Glabella mit gut entwickeltem Frontallobus, der vorn in der Mitte gerade und an den Seiten abgeschrägt ist. Lateralloben leicht nach vorn gerichtet, die dritten am kräftigsten. Präglabellarfeld nicht ausgebildet. Occipitalring gut ausgebildet, Wangenstachel gerade nach hinten gerichtet. Cranidium granuliert bis zart tuberkuliert, ebenso die Freiwangen. Pygidium ist länger als breit, mit vier Rippenpleuren. Rhachis in der Mitte durchgebogen und mit paarig ange- legten Tuberkeln versehen. Hypostom und Thorax nicht bekannt.

Beschreibung: Cranidium flach gewölbt, Festwangen etwas mehr gewölbt und zu den Wangenstacheln steiler abfallend. Lateralloben gut entwickelt und leicht nach vorn gerichtet. Erste Loben buckelförmig, vom Occipitalring durch tiefe Gruben getrennt und von den zweiten Loben, die etwas schlanker sind, durch tiefe, dreieckige Lateralfurchen abgegrenzt. Die dritten Loben etwas kräftiger, flach und breit; von den zweiten durch tiefe, ovale Lateralfurchen abgesetzt und von dem gut ausgebildeten Frontallobus mit mittelgroßer, tiefer, dreieckiger Grube, die vorn von der schräg nach hinten gerichteten Augenleiste begrenzt wird. Frontallobus vorn, in der Mitte gerade, zu den Seiten abgeschrägt. Präglabellarfeld nicht ausgebildet. Die Schräge setzt sich im Innenteil der Gesichtsnaht, die über die Augenstiele bogenförmig nach außen zur Wangenstachelbasis verläuft, fort.

Occipitalring breit, durch eine weiche, bogenförmige, nach vorn gerichtete Furche abgesetzt und mit drei großen, flachen Tuberkeln versehen. Vermutlich war der Mediantuberkel als kleiner Stachel ausgebildet. Dorsalfurchen deutlich ausgeprägt und die Glabella zu den Festwangen abgrenzend. Festwangen breit, hinten gerade, durch eine breite, tiefe Hinterrandfurche, die bis zur Wangenstachelbasis gerade bleibt, vom Hinterrand getrennt, um dann in einem Bogen nach vorn in der Gesichtsnaht zu enden. Hinterrand schmal, in Höhe der Augen in weichem Bogen nach hinten verlaufend, deutlich breiter werdend und dann übergehend in gerade nach hinten gerichtete Stachel, die ungefähr die Länge der Glabella erreichen (Tafel 4: Figs 5-7 in Nikolaisen, 1961.; Tafel 3: 7-8 sowie Abb. 6 diese Arbeit).

Freiwangenfeld dreieckig, leicht gebläht, Außenrand bogenförmig, vorn in ein kleines Pseudoglabellarfeld übergehend (Tafel 4: Fig. 8 in Nikolaisen, 1961; Tafel 3: 9 diese Arbeit). Oberfläche des Canidiums mit kleinen bis mittleren, flachen Tuberkeln überzogen. Pygidium triangulär, etwas länger als breit, mit vier Rippenpleuren, von denen die ersten beiden bogenförmig nach hinten verlaufen und die beiden inneren parallel zur Rhachis nach hinten gerichtet sind. Die Enden laufen in feine Spitzen aus, wobei die beiden äußeren leicht nach außen gerichtet sind. Auf den Pleurenrippen vereinzelt große Tuberkeln. Mittelteil der Rhachis leicht durchgebogen und mit vier bis fünf paarig angeordneten Tuberkeln besetzt. Rhachis in ungefähr zwanzig 


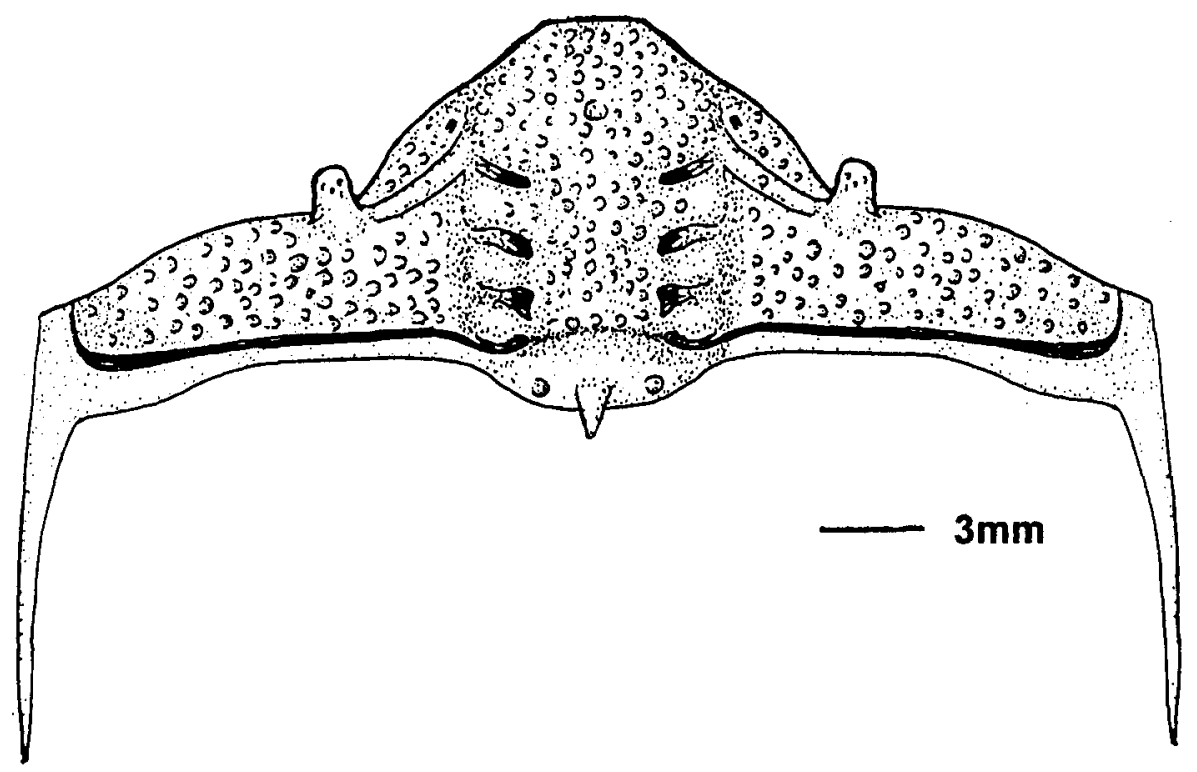

Abb. 6. Rekonstruktion von Atractocybeloides gracilis (Nikolaisen, 1961).
Seitenrippen gegliedert (Tafel 4: Fig. 9 in Nikolaisen, 1961).

Unterschiede: Atractocybeloides gracilis ist von den bis jetzt aus Baltoskandia bekannten Arten die jüngste Spezies. Sie unterscheidet sich deutlich von den älteren Vertretern durch ihre relativ glatte Oberfläche, die nur schwach granuliert und mit kleinen bis mittleren flachen Tuberkeln bedeckt ist.

Vorkommen: Von Nikolaisen (1961) wurde diese Art unter Atractopyge gracilis aus dem Oslo-Gebiet aus dem oberen Chasmops-Kalk $4_{b \delta}$ von Terneholmen Asker beschrieben. Stratigraphisch kann die Art in die Rakvere-Stufe E von Baltoskandia gestellt werden. Die Rakvere-Stufe ist als Geschiebe durch die sehr feinen und dichten Ostseekalke im ganzen Vereisungsgebiet südlich der Ostsee vertreten.

\section{Schlussfolgerungen}

Die Gattung Atractocybeloides, die zur Subfamilie Cybelinae gehört, ist eine kleinwüchsige Form, die mit zwei Arten, A. berneri und A. vonhachti, 1991 neu aufgestellt wurde. Beide Arten waren nur in wenigen Resten bekannt und ließen sich durch ihre Begleitfauna in die Idavere- bis Keila-Stufe einordnen.

In den letzten zehn Jahren sind weitere Panzerreste von $A$. berneri und $A$. vonhachti aus der reichhaltigen Sammlung Rhebergen hinzugekommen, so daß eine Rekonstruktion des Panzers beider Arten möglich war. Außerdem erweitert sich das Artenspektrum um die beiden neuen Arten $A$. nebeni n. sp. aus dem Folkeslunda-Kalk der Lasnamägi-Stufe $\mathrm{C}_{\mathrm{Ib} \gamma}$ und $A$. oepiki n.sp. aus dem Kuckersit der Kukruse-Stufe $\mathrm{C}_{\mathrm{II} \alpha}$ (Abb. 1). A. nebeni n. sp. ist derzeit die älteste Art dieser Gattung. Die von Nikolaisen (1961) neu beschriebene Art Atractopyge gracilis, die aus dem oberen Chasmops-Kalk $4_{b \delta}$ stammt, ist bis jetzt die jüngste Art dieser Gattung (Abb. 1). Der Chasmops-Kalk ist gleichzusetzen mit den Schlammkalken (Ostseekalken) der RakvereStufe E. Durch ihr seltenes Vorkommen und ihre geringe Größe wurde diese Gattung erst vor zehn Jahren entdeckt.

Es bestehen Ähnlichkeiten zur nordamerikanischen, ebenfalls kleinwüchsigen Gattung Cybeloides.

\section{Danksagung}

Der Autor dankt Dr. M. Aberhan, Dr. Geyer und Herrn Dr. R. Schallreuter für ihre Hilfe bei der Fertigstellung des Manuskripts.

\section{Literatur}

Chatterton , B. D. C. \& Ludvigsen, R. 1976. Silicified Middle Ordovician trilobites from the South Nahanni River Area, District of Mackenzie, Canada. - Palaeontographica (A) 154 (1/3): 1-106.

Evitt, W. R. \& Tripp, R. P. 1977. Silicified Middle Ordovician trilobites from the families Encrinuridae and Staurocephalidae. - Palaeontographica (A) 157 (4/6): 109-174.

Hucke, K. \& Voigt, E. 1967. Einführung in die Geschiebeforschung (Sedimentärgeschiebe). - 132 S., Oldenzaal (Nederlandse Geologische Vereniging).

Jaanusson, V. 1960. The Viruan (Middle Ordovician) of Öland. - Bulletin of the Geological Institutions of the University of Uppsala 38 (3/4): 207-288.

- 1965. Lower and Middle Viruan (Middle Ordovician) of the Siljan District. - Bulletin of the Geological Institution of the University of Uppsala 42: $40 \mathrm{~S}$. 
Krueger, H.-H. 1991. Die neue ordovizische Trilobitengattung Atractocybeloides mit zwei neuen Arten aus baltoskandischen Geschieben. - Archiv für Geschiebekunde 1 (3/4): 225-230.

Kummerow, E. 1927. Beiträge zur Kenntnis der Fauna und der Herkunft der Diluvialgeschiebe. - Jahrbuch der PreuBischen geologischen Landesanstalt 48 (1): 1-59 (Band 1928).

Moore, R. C. (ed.) 1959. Treatise on Invertebrate Paleontology, Part O (Harrington, H. J., Henningsmoen, G. et al.: Arthropoda 1 (Arthropoda - General Features Proarthropoda Euarthropode - General Features Trilobitomorpha)). - XIX + 560 S., Lawrence, Kans./New York, N.Y. (Geological Society of America/University of Kansas).

Neben, W. \& Krueger, H.-H. 1971. Fossilien ordovicischer Geschiebe. - Starinigia 1:1-7.

Nikolaisen, F. 1961. The Middle Ordovician of the Oslo region, Norway, 7. Trilobites of the Suborder Cheirurina. Reprint from Norsk Geologisk Tidskrift 41 (2-4): 279-310, Bergen 1961.

Öpik, A. 1925. Beiträge zur Kenntnis der Kukruse-( $\mathrm{C}_{2}$-)Stufe in Eesti I. - Acta et Commentationes Universitatis Dorpatensis (A) 8 (5): $20 \mathrm{~S}$. = Publications of the Geological Institution of the University of Tartu $4 b$.

- 1937. Trilobiten aus Estland. - Acta et Commentationes Universitatis Tartuensis (Dorpatensis) (A) 32 (3): $163 \mathrm{~S}$.

Pompeckj, J. F. 1890. Die Trilobiten-Fauna der Ost- und Westpreussischen Diluvialgeschiebe - Beiträge zur Naturkunde Preussens 7: I+97 S. (Inaugural-Dissertation Albertus-Universität zu Königsberg in Pr. (R. Leupold)).

Roomusoks, A. 1970. Stratigraphy of the Viruan Series (Middle Ordovician) in Northern Estonia. - 348 S. Tallinn (Valgus).

Törnquist, S. L. 1884. Undersökningar öfver Sijansomradets trilobitfauna - Sveriges Geologiska Undersökning (C) 66: 101 S., Stockholm.
Schmidt, F. 1881. Revision der Ostbaltischen Silurischen Trilobiten nebst Geognostischer Übersicht des Ostbaltischen Silurgebietes, Abt. 1, Phacopiden, Cheiruriden und Encrinuriden. - Mémoires l'Académie Impériale des Sciences de St.-Pétersbourg (7) 30 (1): $237 \mathrm{~S}$

Shaw, F. C. 1968. Early Middle Ordovician Chazy trilobites of New York. - New York State Museum, Memoirs 17: $114 \mathrm{~S}$.

Skupin, K.; Speetzen, E. \& Zandstra, I. G. 1993. Die Eiszeit in Nordwestdeutschland. - Geologisches Landesamt Nordrhein-Westfalen: 143 S., Krefeld.

Slocom, A. W. 1913. New trilobites from the Maquoketa Beds of Fayette County, Iowa. - Field Museum of Natural History 4 (3): 43-83.

Tripp, R. P. 1980. Trilobites from the Ordovician Ardwell Group of the Craighead Inlier, Girvan district, Scotland - Transaction of the Royal Society of Edinburgh (Earth Science) 71: 123-145.

Vogdes, A. W. 1890. A bibliography of Palacozoic Crustacea from 1698 to 1889, including a list of North American species and a systematic arrangement of genera. - Bulletin of the U.S. Geological Survey 63: 177 S.

Warburg, E. 1925. The Trilobites of the Leptaena Limestone in Dalarne. With a Discussion of the zoological Position and the Classification of the Trilobita. - Bulletin of the Geological Institution of the University of Upsala 17: I-VI + 1-446.

Wigand, G. 1888. Ueber die Trilobiten der silurischen Geschiebe in Mecklenburg. - Zeitschrift der deutschen geologischen Gesellschaft 40 (1): 39-101.

Wiman, C. 1908. Studien über das nordbaltische Silurgebiet II. - Bulletin of the Geological Institution of the University of Upsala 8: $73-168$. 